\title{
Selection rules for bimolecular photoabsorption
}

\author{
David L. Andrews and Alexander M. Bittner \\ School of Chemical Sciences, University of East Anglia, Norwich NR4 7TJ, UK
}

Received 24 February 1992

\begin{abstract}
Bimolecular photoabsorption is a term describing processes in which two molecules or other chemically distinct entities undergo simultaneous excitation to electronic excited states, the energy being provided by the absorption of one or more laser photons. These processes have previously been referred to as synergistic, cooperative, or mean-frequency absorption. This paper introduces a new terminology for the description of such phenomena and provides an overview of the features associated with bimolecular processes involving the absorption of one, two or three photons from a single laser beam. Emphasis is placed on a detaled symmetry analysis based on irreducible tensors, leadıng to a comprehensive treatment of the selection rules for all the major molecular point groups. Finally, attention is drawn to some systems in which these effects have been detected, and others in which they might be expected to occur.
\end{abstract}

\section{Introduction}

The absorption of one, two or more photons leading to the simultaneous excitation of two centres (atoms or molecules) may be termed bimolecular photoabsorption. Such processes, which have elsewhere been referred to as synergistic, cooperative or collective, are better described by the more informative adjective bimolecular, which avoids possible connotations of many-body phenomena. Subject to appropriate selection rules, bimolecular photoabsorption occurs through an exact cancellation of energy mismatches between the photon energy supplied and the energy uptake by each participating atom or molecule, subject to overall energy conservation by each pair. In any such process, none of the molecular transition energies should match any of the photon frequencies or their multiples, because then simple one-centre transitions would occur, selection rules again permitting.

In this paper bimolecular single colour $n$-photon absorption processes, i.e. those which can be induced by a single laser beam, are considered. Cases involving the absorption of up to three photons are ana-

Correspondence to: D.L. Andrews, School of Chemical Sciences, University of East Anglia, Norwich NR4 7TJ, UK. lysed in detail. The following equation generally depicts the excitation process:

$\mathrm{A}+\mathrm{B}+n \hbar \omega \rightarrow \mathrm{A}^{*}+\mathrm{B}^{*} \quad(n=1,2,3, \ldots)$.

Figs. 1-3 depict the energy levels involved in one-, two- and three-photon excitation, respectively. It is clear that in two-photon (mean-frequency) bimolecular absorption (fig. 2) one should avoid the case of two isoenergetic excitations, as one-photon absorption - if allowed - would again dominate. However, $A$ and $B$ can designate two different molecules of the same chemical species if the process leads to two different excited states as assumed in fig. 2. For the oneand three-photon cases no such distinction has to be drawn, i.e. the labels A and B may refer to molecules

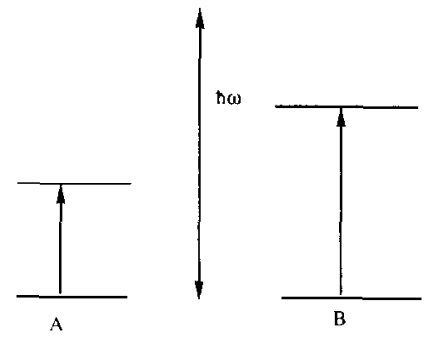

Fig. 1. Energy levels of molecules A and B involved in one-photon bimolecular absorption. 


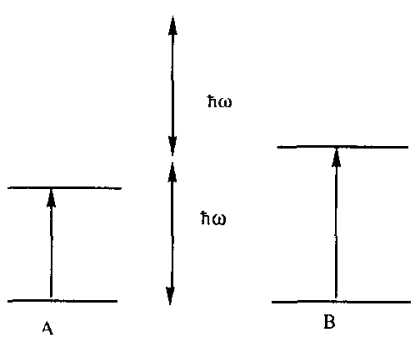

Fig. 2. Energy levels of molecules A and B involved in two-photon bimolecular absorption.

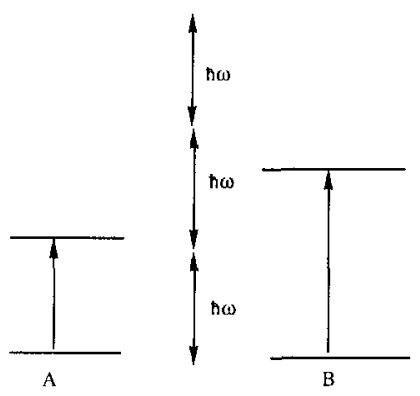

Fig. 3. Energy levels of molecules A and B involved in three-photon bimolecular absorption.

of the same species and the final states may even be the same. In passing we note that for (multi-photon) bimolecular emission eq. (1) would apply with a reversed arrow.

It should be emphasised that the processes of interest here are not to be regarded as collisional. Numerous experimental studies have demonstrated the occurrence of bimolecular excitation, both involving single- and two-photon absorption; recent examples are afforded by studies of media such as van der Waals molecules [1], liquids [2], solids [3,4] or in matrixisolated species [5]. In all these cases it is clear that it is the proximity of the participating species, rather than their collision, which induces the absorption, despite the observation in certain systems of similar effects in the gas phase. In this connection we can also note that there is a closely related phenomenon usually known as laser-induced collisional excitation transfer (LICET) where one of the centres is already in an excited state:

$$
\mathrm{A}^{*}+\mathrm{B}+n \hbar \omega \rightarrow \mathrm{A}+\mathrm{B}^{*} \quad(n=1,2,3, \ldots) .
$$

Bimolecular photoabsorption may play a role in photosynthetic energy transfer; however in order to elucidate the underlying mechanism it is useful for current theory and experiment to restrict the scope to small systems. All such processes can be described in a unified way as described in section 2 .

One of the earliest and classic experiments on bimolecular excitation studied two-photon absorption by a gaseous mixture of thallium and barium [6]. Much of the subsequent experimental work has dealt with collision-induced absorption [7] and LICET in gases [8], and cooperative effects in solids containing rare earth ions [3]. Recent experiments have mostly focused on one- and two-photon excitation of noble gas-molecular halogen van der Waals complexes $R \ldots X_{2}$ [5,9]. The resultant electron transfer and reaction to form a noble gas-halogen exciplex $\mathrm{R}$ $\mathrm{X}^{*}$ provides a good method for detection (via exciplex fluorescence). Three-photon bimolecular processes have to our knowledge not yet been observed.

In the following section a simplified representation of the theory, based on irreducible Cartesian tensors, is presented. Various features of the two-photon case, such as its resonance and polarisation behaviour, have been detailed in a recent review [10]; however none of the processes to be considered have previously been the subject of an irreducible tensor analysis. The tensor formulation enables the general features of $n$ photon bimolecular absorption to be identified without the complexity of the detailed quantum calculations, which arc presentcd elsewhere [11-15]. In section 3 the selection rules for atoms and for molecules of common point groups are derived, and the results for $n=1,2$ and 3 are presented in an easily applicable table. In section 4 our results are assessed in the light of the experiments mentioned above. New experiments aimed at confirming both the occurrence and the mechanism of one-, two- and threephoton bimolecular absorption processes are suggested, and physically realistic examples are given.

\section{Tensor representation}

The detailed theoretical representation of bimolecular photoabsorption processes requires application of well-established quantum electrodynamical methods, based on electric dipole coupling with radiation. 
However for the purposes of this paper it is sufficient to consider a simplified approach which retains the features essential to recovery of the proper selection rules. The quantum electrodynamical theory of the two-photon case has been treated in detail elsewhere [16]; the formulation of the one- and three-photon cases follows along broadly similar lines and will be detailed in a subsequent paper [15].

To facilitate the development of the necessary tensor formulation, it is useful to introduce diagrams which display the radiative interactions involved in each of the processes under consideration. It should first be noted that the energy mismatch is conveyed from one molecule to the other by means of virtual photon coupling, leading to a dependence of the rate on the inverse sixth power of the intermolecular distance (in the short-range limit) [16]. Conscquently bimolecular one-photon absorption can be represented by the diagram shown in fig. 4 , in which it is arbitrarily assumed that the real (laser) photon is absorbed by molecule $A$. In this case the virtual photon has to carry over the entire excitation energy to molecule B. Fig. 4 embodies the characteristics of the set of six time-ordered diagrams which is involved in the more formal representation of the process. In passing we note that although three of those diagrams entail emission of the virtual photon at $A$ and its absorption at $B$, three others involve propagation from $B$ to $A$; consequently no arrowhead is placed on the wave connecting $\mathrm{A}$ to $\mathrm{B}$ in fig. 4.

In the two-photon case there are two possible mechanisms, illustrated by figs. $5 \mathrm{a}$ and $5 \mathrm{~b}$. In the former, both laser photons are absorbed by molecule $\mathrm{A}$ and the entire excitation energy for $B$ is conveyed by the virtual photon. In the latter, each molecule absorbs one laser photon and the virtual photon mediates exchange of the energy mismatch. These two mechanisms have previously been referred to as distributive and cooperative, respectively [10]. Equally,

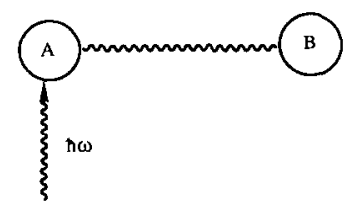

Fig. 4. Diagram representing one-photon bimolecular absorption, corresponding to the partition $(2,1)$ (see text).

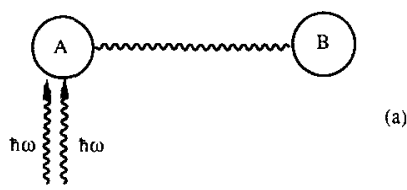

(a)

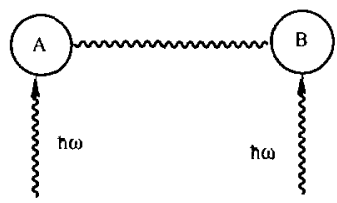

(b)

Fig. 5. Diagrams representing two-photon bimolecular absorption; (a) corresponds to the partition $(3,1)$, known as the distributive mechanism; (b) corresponds to the partition $(2,2)$, known as the cooperative mechanısm (see text)
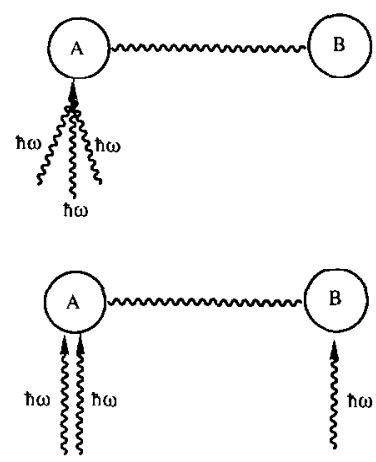

(b)

Fig. 6. Diagrams representing three-photon bimolecular absorption; (a) corresponds to the partition $(4,1)$; (b) corresponds to the partition $(3,2)$ (see text).

in the three-photon case there is the possibility of either all three photons being absorbed by one molecule, as illustrated in fig. $6 \mathrm{a}$, or one molecule absorbing two photons and the other one photon, as in fig. 6(b). As for the one-photon case, similar arguments concerning the directions of propagation of the virtual photon apply to the two- and three-photon cases.

In passing it is worth considering the analysis of the general case involving $n$-photon bimolecular absorption. Each of the $n$ laser photons may be absorbed at either of the participating molecules, the various possibilities corresponding to the two-fold partitions of $n$. For example the partition $\{2, n-2\}$ represents the case where two photons are absorbed at $\mathrm{A}$ and $n-2$ at $\mathrm{B}$. The virtual photon emission and absorption interactions must occur at different 
centres, so that in the above example there are in total three interactions at $\mathrm{A}$ and $n-1$ at $\mathrm{B}$, giving the partition $(3, n-1)$. In the general case, therefore, the possibilities correspond to the two-fold partitions $(p, q)$ of $n+2$, subject to constraints $p \geqslant 1, q \geqslant 1$.

It is proposed to refer to these partitions to unambiguously identify the various mechanisms for bimolecular photoabsorption. Their virtue is that they directly signify the selection rules associated with the transitions at the two participating centres. For example the mechanism known as cooperative twophoton absorption will be referred to as the $(2,2)$ mechanism (two-photon selection rules at each centre), and the distributive mechanism will be referred to as the $(3,1)$ mechanism (three-photon at one centre and one-photon at the other). In general it is to be assumed that wherever a mechanism involves a different number of interactions at the two centres, $\mathrm{A}$ is defined as the molecule where the larger number of interactions occurs.

The tensor formulation of any mechanism relating to a partition $(p, q)$ involves a molecular response tensor $T_{p}$ of rank $p$, with $3^{p}$ components, for molecule $\Lambda$ and a tensor $T_{q}$ of rank $q$, with $3^{q}$ components, for B. Each tensor may have index symmetry properties which reduce the number of its independent components to less than $3^{p}$ or $3^{q}$, however. For molecule A, the tensor $T_{p}$ represents one virtual photon interaction and $p-1$ laser photon interactions, and therefore possesses permutational symmetry in $p-1$ of its indices. Using terminology introduced clscwhere [17], this index symmetry can be signified by writing $T_{p}$ as $T_{1(p-1)}$. Similarly the index symmetry properties of the molecular response tensor for molecule B can be represented by $T_{1(q-1)}$.

The rate $\Gamma$ of bimolecular photoabsorption can now be expressed in terms of the above tensors and a coupling tensor $V_{2}$ which represents the retarded resonance electric dipole-electric dipole interaction [18]

$\Gamma \propto X_{n} \odot^{n} \bar{X}_{n}$,

where

$X_{n}=\sum_{p=1}^{n+1} T_{1(p-1)} \odot V_{2} \odot T_{1(n+1-p)}$.

Here the symbol $\odot$ represents a tensor contraction, i.e. a summation over paired indices as in the scalar product between two vectors. Each of the terms in eq.
(4) represents a contribution associated with a different mechanism $(p, q)$, where $q=n+2-p$.

In general each tensor $T$ will transform under a reducible representation of the symmetry point group for its parent molecule. As such, it is convenient in the analysis of selection rules to express each tensor as a sum of irreducible tensors of weight $j$ [19]. These can be represented as $T_{1(p-1)}^{()}$with $0 \leqslant j \leqslant p$ and $T_{\text {I(q-1) }}^{()}$with $0 \leqslant j \leqslant q$. One other feature worth noting is that for $p, q=1$ or 3 , weight -0 contributions vanish [17]. Since only electric dipole coupling is considered here, parity labels are left implicit, although they are crucial in determining the selection rules, since they are simply given by $(-1)^{p, q}$. Further details are given in the appendix.

\section{Selection rules}

Using the results of the last section, it is now possible to derive the selection rules which govern the excited states which can be accessed in bimolecular photoabsorption. In table 1 , the irreducible representations of the allowed final states, for both molecules $\mathrm{A}$ and $\mathrm{B}$, are listed for the common molecular point groups. These selection rules are designed for application to cases in which the transitions in both molecules originate from ground states possessing the full symmetry of the corresponding molecules. Where cither ground statc does not belong to the totally symmetric representation, the tabulated selection rules apply to the direct product of the ground and excited state representations.

The table is divided into five columns; the first relates to one-photon bimolecular absorption, the next pair of columns to the two mechanisms for the twophoton process and the last pair to the two mechanisms for the three-photon case. In general, any given mechanism can take place only when representations of both $A$ and $B$ are ticked in the appropriate column. For any pair of non-identical molecules, it is of course necessary to consider the two possibilities of applying the labels $A$ and $B$. The principles of applying table 1 are best illustrated by reference to a number of specific examples. In the following, various hypothetical molecules of given symmetries are considered simply to clarify application of the selection 
Table 1

Selection rules for bimolecular photoabsorption in molecules of the common point groups with mechanisms denoted by the partitions $(p, q)$. For completeness the spherical group $\mathrm{K}_{\mathrm{h}}=\mathrm{K} \otimes \mathrm{S}_{2}$, where $\mathrm{K}$ [SO(3)] is the full rotation group, is included for application to free atoms and ions. Where a representation is not shown, it is forbidden in all cases

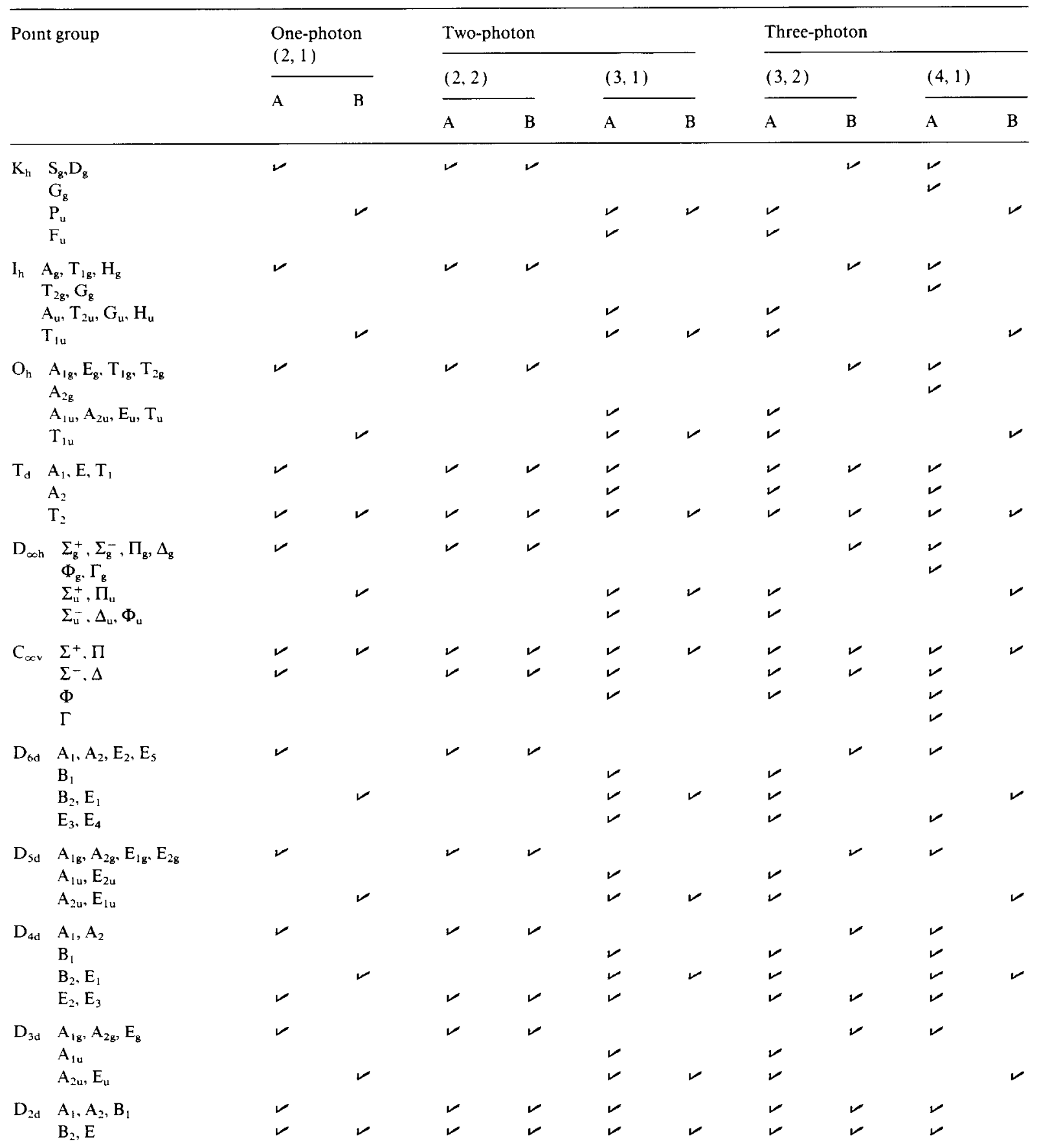


Table 1 (continucd)

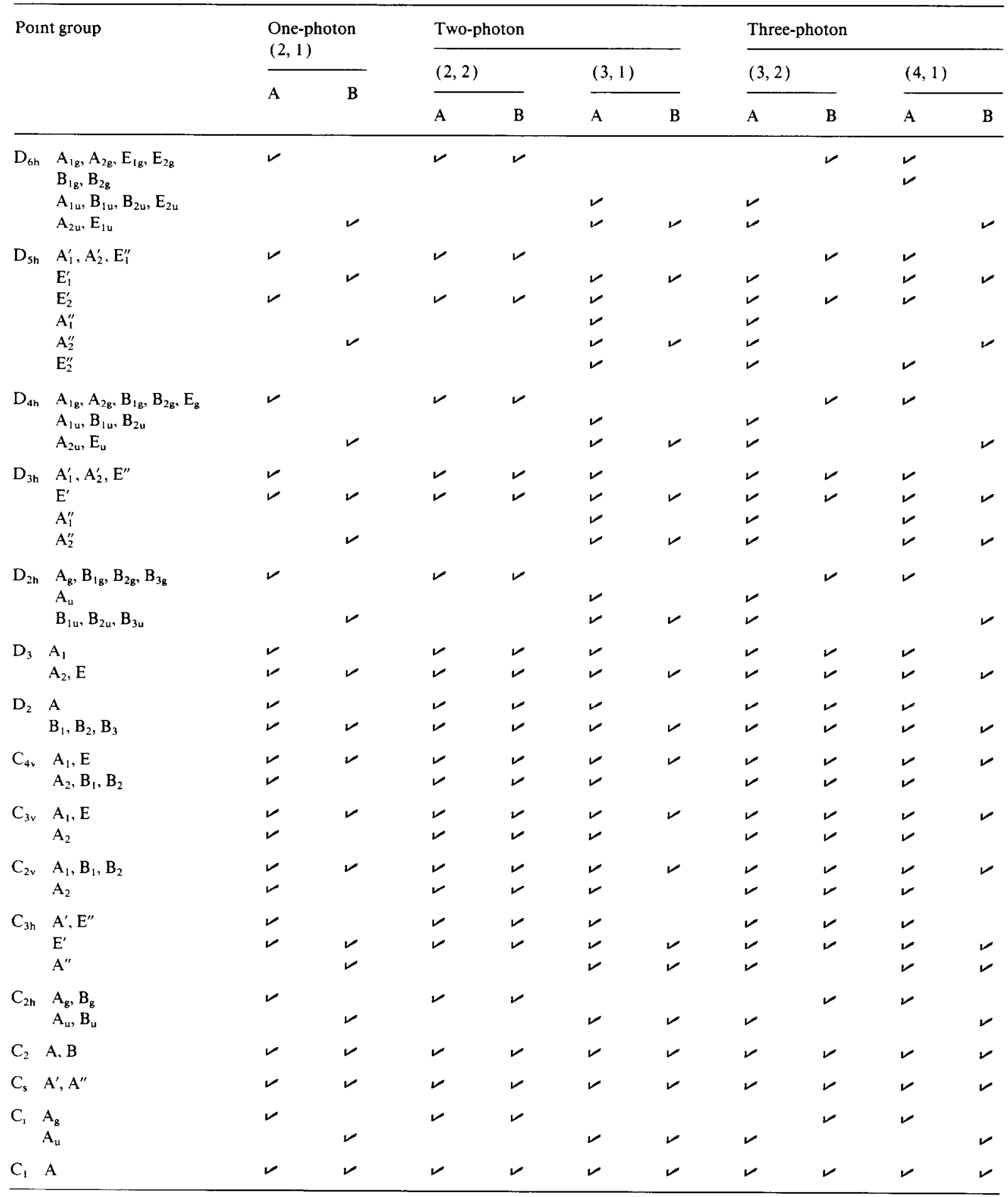


rules. Examples involving actual compounds are subsequently discussed in section 4 .

Consider first the case of one-photon excitation. Let us assume that one molecule is octahedral (point group $\mathrm{O}_{\mathrm{h}}$ ) and the other belongs to the point group $\mathrm{C}_{1}$. Suppose that the octahedral molecule is labelled $A$ and its partner $B$. Inspecting the $\mathrm{O}_{h}$ entries under the heading $\mathrm{A}$ in the one-photon column shows that the excitation (from an $\mathrm{A}_{\mathrm{lg}}$ ground state) might be to either an $A_{1 g}, E_{g}, T_{1 g}$ or a $T_{2 g}$ excited state. Applying the same principle to $B$, by reference to the $C_{1}$ entries under the heading $B$ in the one-photon column, reveals that only a transition to an $A_{u}$ state is allowed. Conversely if the labels $A$ and $B$ are exchanged, it is clear that one other possibility is a transition to an $\mathrm{A}_{\mathrm{g}}$ state in the $\mathrm{C}_{1}$ molecule, accompanied by a transition to a $T_{1 \mathrm{u}}$ state in the octahedral species.

A slightly more complex example of the one-photon process is afforded by a case where a $C_{2 v}$ molecule is excited from $a B_{2}$ to $a B_{1}$ state, the direct product symmetry being $A_{2}$. This satisfies the selection rules for $A$, according to the $C_{2 v}$ entries in the first column. If a counterpart molecule $B$ belongs to the $T_{d}$ point group and has a ground state $A_{1}$, inspection of the table shows that the only accessible excited state symmetry is $T_{2}$. Because the direct product symmetry of the transition in the $C_{2 v}$ molecule is $A_{2}$, the possibility of it acting as $B$ does not arise. Since this molecule has to carry the label $\mathrm{A}$ in this case, it indicates that the single laser photon must be absorbed there, rather than at the tetrahedral site.

An instructive example for the two-photon bimolecular process is where $\mathrm{A}$ and $\mathrm{B}$ are two chemically different molecules belonging to the point group $\mathrm{C}_{\infty}$, and where both are excited from $\Sigma^{+}$to $\Delta$ states. Ticks appear for both $\mathrm{A}$ and $\mathrm{B}$ only in the column $(2,2)$ so that only this, not the $(3,1)$ mechanism, can apply. This means that each molecule must absorb one laser photon.

Finally, let us assume that on bimolecular absorption of three photons a $D_{4 h}$ molecule with ground state $A_{1 g}$ enters an $E_{u}$ state and $a D_{6 h}$ molecule with ground state $A_{1 \mathrm{~g}}$ an $E_{2 \mathrm{~g}}$ state. If the first is labelled $A$ and the latter $B$, only column $(3,2)$ qualifies. With opposite labelling, only column $(4,1)$ qualifies. The conclusion is that both mechanisms are possible here.

\section{Discussion}

For molecules separated by typical nearest-neighbour distances, it has been estimated that the rate of bimolecular $n$-photon absorption approaches that of $n$-photon absorption by individual molecules [10]. Such processes can also exhibit near-resonance enhancement given excited states of suitable energies and symmetry properties; then bimolecular processes involving molecules at larger distances, such as in the gas phase, become observable. It should also be noted that if the excited levels of the participating molecules are not low in energy one would have to employ UV or even VUV radiation in the one-photon case. Here it may be experimentally more feasible to utilise two-photon excitation.

It is worth re-emphasising that no overlap of wavcfunctions between the initial (ground state) entities need be present. For this reason, and also because the states which are formed by these processes entail excitation of both centres, our formalism can appropriately be applied to a wide range of chemical systems, including the van der Waals molecules whose known excited states are usually described in terms of MO theory.

\subsection{Related experiments}

A few examples of a bimolecular photoabsorption have been observed and are now well established in the literature. In particular, one-photon bimolecular excitation processes have been widely studied in rare earth ion-doped crystals. An example is provided by the process $2 \mathrm{~Tb}^{3+}\left({ }^{7} \mathrm{~F}_{6}\right)+h \nu \rightarrow \mathrm{Tb}^{3+}\left({ }^{5} \mathrm{D}_{4}\right)+$ $\mathrm{Tb}^{3+}\left({ }^{7} \mathrm{~F}_{2}\right)$ which occurs in $\mathrm{Tb}^{3+}$ doped $\mathrm{CaWO}_{4}[3]$. The direct products of the symmetry representations of the rotation group $K_{h}$ contain $P_{u}$ and $D_{g}$, which satisfy the selection rules listed in table 1 . In passing we note that such one-photon transitions appear to have the character of dipole-quadrupolc coupling. Gudzenko and Yakovlenko first predicted this selection rule for one-photon biatomic processes in 1972 [20].

In another solid-state example, the two-photon absorption $2 \mathrm{Nd}^{3+}\left({ }^{4} \mathrm{I}_{11 / 2}\right)+2 h \nu \rightarrow 2 \mathrm{Nd}^{3+}\left({ }^{4} \mathrm{G}_{7 / 2}\right)$ has been observed in $\mathrm{Nd}^{3+}$ doped $\mathrm{LaCl}_{3}$ [4]. Applying the direct product rule and referring to tablc 1 shows that the $(2,2)$ mechanism alone should apply. The 
excited states are isoenergetic but the corresponding one-photon single ion transition is forbidden. This is the reason why the chemical equation written above to describe the excitation cannot simply be divided by 2 . In these experiments, and in many others, bimolecular absorption has been detected without any involvement of near-resonance enhancement.

A classic case concerns the two-photon excitation of a gaseous mixture of barium and thallium, observed by White in 1981 using a tunable dye laser [6]. The barium excitation is $6 \mathrm{~s}^{2}{ }^{1} \mathrm{~S}_{0} \rightarrow 6 \mathrm{~s} 6 \mathrm{p}{ }^{1} \mathrm{P}_{1}$ (representation $P_{u}$ in the rotation group $K_{h}$ ), the thallium route $6 p^{2} P_{1 / 2} \rightarrow 7 s^{2} S_{1 / 2}$ (same representation). Using table 1 it is clear that the process is only two-photon allowed and that the $(3,1)$, not the $(2,2)$, mechanism should operate. It is worth noting that thallium possesses a near-resonant $9 \mathrm{p}^{2} \mathrm{P}_{3 / 2}$ state $25 \mathrm{~cm}^{-1}$ above $2 h \nu$; according to the appropriate tensor $T_{1(2)}$ this should lead to near-resonance enhancement. This may explain why it proved possible to observe the effect with the relatively low laser flux of $\approx 6.5 \times 10^{12}$ $\mathrm{W} \mathrm{m} \mathrm{m}^{-2}$.

Observations in the gas phase such as that discussed above are often regarded as collision-induccd; however the term is clearly inapplicable to some of the other examples like ion-doped crystals and the processes to be examined below. It is worth emphasising that although such bimolecular transitions occur with greatest efficiency when the intermolecular distance $R$ is very small, owing to the $R^{-6}$ range dependence, it is nonetheless essentially a proximity effect. However in the processes we describe there is no requirement for the electron distributions of the participating species to overlap; also there is no need for involvement of collisional kinetic energy.

Many experiments deal with one- and two-photon excimer-pumped dye laser-induced reactions $\mathrm{R}+$ $\mathrm{XY}+n h \nu \rightarrow \mathrm{R}^{+} \mathrm{X}^{-}+\mathrm{Y} \rightarrow \mathrm{RX}^{*}+\mathrm{Y}$ ( $\mathrm{R}$ denotes a rare gas, $X$ and $Y$ halogens) in the gas phase $[1,9]$, in rare gas.liquids [2] or matrices [5]. It is difficult to apply the selection rules here, although the charge transfer transition can be identified with the symmetry of a translation along R...X. At any rate, enhancement associated with the approach to resonance at $h \nu$ seems to be important again, at least in the case of $\mathrm{Kr}+\mathrm{F}_{2}$ [21].

In this context we note that Last and George have developed a theory for one-photon absorption and subsequent electron transfer in solids [22]. The similarities to our approach consist of their assumption that no wavefunction overlap should be present and that the absorption rate (which is proportional to the square of the overall transition moment) decreases with the sixth power of the intermolecular distance.

\subsection{Suggested experiments}

Whereas most of the experimental studies referred to above involve simple atoms or ions, or at most diatomic species, there are a number of systems involving larger molecules in which we suggest that it should be possible to observe bimolecular absorption. The selection rules which apply in such cases are in many respects more interesting. Using table 1 and restricting the scope to highly symmetrical molecules we suggest the following experiments, dealing first with cases where only one species is involved (i.e. $A=B$ ).

The tetrahedral molecule $\mathrm{RuO}_{4}$ absorbs at 26000 $\mathrm{cm}^{-1}\left({ }^{1} \mathrm{~A}_{1} \rightarrow{ }^{1} \mathrm{~T}_{2}\right)$ in the gas phase $[23,24]$ and should in the liquid or solid state be a very suitable candidate for a one-photon experiment: the $\mathrm{RuO}_{4}$ entities csscntially retain their clcctronic integrity in the crystal and intermolecular interactions are weak [25]. There is very little conventional absorption at 52000 $\mathrm{cm}^{-1}[23,24]$ and this wavenumber corresponds to the $192.3 \mathrm{~nm}$ wavelength available from the powerful argon fluoride exciplex lasers. However, the compound exhibits a complex photochemistry [26].

Solid tetrahalogenocobaltate (II) salts give rise to two absorptions, ${ }^{4} \mathrm{~A}_{2} \rightarrow{ }^{4} \mathrm{~T}_{1}(\mathrm{~F})$ and ${ }^{4} \mathrm{~A}_{2} \rightarrow{ }^{4} \mathrm{~T}_{1}(\mathrm{P})$ in the point group $T_{d}$, at $\approx 5000$ and $14000 \mathrm{~cm}^{-1}$; the exact wavenumbers depend on the halogen chosen [2730 ]. As shown in table 1 , all mechanisms relating to the one-, two- and three-photon cases are allowed, so a wide range of experiments can be designed. An example is provided by crystalline bis (tetramethylammonium) tetrabromocobaltate(II) [28] where a one-photon absorption should excite the ${ }^{4} T_{1}(F)$ level in one anion and the ${ }^{4} \mathrm{~T}_{1}(\mathrm{P})$ level in a neighbouring anion. It should occur at the second harmonic of a Nd: YAG laser.

The most convincing candidates for bimolecular photoabsorption will be found using three-photon excitation of virtually non-interacting atoms. The $\mathrm{Be}_{2}$ dimer (formed in the gas phase) [31] is a small but very loosely bound species (van der Waals potential 
$800 \mathrm{~cm}^{-1}$, interatomic distance $250 \mathrm{pm}$ ) [32]. Exciting a $2 p^{1} P_{1}$ level in one atom and a $3 s^{1} S_{0}$ level in the other should be accomplished by three photons $(308.5 \mathrm{~nm})$. Here a xenon chloride laser could provide the necessary wavelength.

A more speculative example is afforded by the $\mathrm{C}_{60}$ "buckminsterfullerene". It has very high symmetry $\left(I_{h}\right)$ and the molecules rotate in the crystal [33], hence little interaction between them is present. By reference to the $n$-hexane solution spectra [34] it can be deduced that one photon of $246.2 \mathrm{~nm}$, or three photons of $738.7 \mathrm{~nm}$, radiation could lead to bimolecular absorption populating the $T_{1 \mathrm{~g}}$ and $\mathrm{T}_{1 \mathrm{u}}$ excited states.

It is easy to find more examples in point groups of lower symmetry, especially if the possibility $A \neq B$ is entertained. In the gas phase $\mathrm{Li}_{2}$ shows a $\Sigma_{\mathrm{g}}^{+} \rightarrow \Sigma_{\mathrm{u}}^{+}$ transition at $14068 \mathrm{~cm}^{-1}$ and $\mathrm{NaK}, \Sigma^{+} \rightarrow \Sigma^{+}$at 12140 $\mathrm{cm}^{-1}$ [35]. A $(2,1)$ mechanism should operate at $381.6 \mathrm{~nm}$, where molecule A should be NaK according to table 1 . The corresponding two-photon absorption process, allowed only by the $(3,1)$ mechanism, should also be observable at a wavelength of 763.2 nm.

Finally we note that there are a number of experimental possibilities for identifying bimolecular absorption. Three examples serve to illustrate the diversity of techniques which may be employed. The most obvious concerns the observation of fluorescence, especially in the case of three-photon absorption by atomic pairs where $\mathrm{A}=\mathrm{B}$. Here any direct fluorescence emitted by the excited atoms will occur at a wavelength which equals two thirds that of the pump photons, corresponding in a sense to an unusual noninteger harmonic conversion process. Secondly, in the two-photon excitation of a mixture of $\mathrm{H}_{2} \mathrm{CO}$ and $\mathrm{D}_{2} \mathrm{CO}$ at $30243 \mathrm{~cm}^{-1}(330.7 \mathrm{~nm})$, corresponding to the mean of the $2{ }_{0}^{1} 4_{0}^{3}$ transitions, it has been shown that evolution of carbon monoxide should occur, enabling chemical or mass spcctrometric identification of the process to be performed [10]. Lastly, the bimolecular photoproduction of two triplet molecules in close proximity should lead to unusually rapid fluorescence associated with triplet-triplet annihilation. For example two iodine molecules might undergo a concerted two-photon process at 13765 $\mathrm{cm}^{-1}(726.5 \mathrm{~nm}$, within the tuning range of a Ti:sapphire laser) leading to the simultaneous population of triplet excited states $A^{3} \Pi_{u}$ and $B^{3} \Pi_{u}[35]$.

\section{Appendix}

The reduction of $T_{m}$ generally results in a sum of irreducible tensors, with some weights represented more than once and therefore distinguished by a secondary label $r$. In the case of a tensor without index symmetry, we have

$T_{m}=\sum_{j=0}^{m} T_{m}^{())}$

where

$T_{m}^{()}=\sum_{r=1}^{N(W)} T_{m}^{(u, r)}$

and $N_{m}^{(U)}$ is the multiplicity of weight $J$ in the reduction scheme. Each irreducible tensor $T_{m}^{(. r)}$ possesses $(2 j+1)$ independent components. The multiplicity with which each tensor weight is represented in the reduction can be derived using a recursive scheme due to Gel'fand et al. [36]; the general result is given by the formula [37]

$N_{m}^{())}=\sum_{k}(-1)^{k} \frac{(2 m-3 k-j-2) ! m(m-1)}{(m-3 k-j) !(m-k) ! k !}$,

where $0 \leqslant k \leqslant\left[\frac{1}{3}(m-j)\right]$. As shown elsewhere [38], the above is the coefficient of $x^{m-1}$ in the Taylor series for $F_{m}(x)=\left(1-x^{3}\right)^{m}(1-x)^{1-m}$ with the property $x^{2 m+1} F_{m}\left(x^{-1}\right)=-F_{m}(x)$. Expansion of each side of the identity gives

$F_{m}(x)=\sum_{j=0}^{m} N_{m}^{(j)}\left(x^{m-j}-x^{m+j+1}\right)$.

Differentiating both this and the defining expression for $F_{m}(x)$ and evaluating $F_{m}^{\prime}(1)$ reveals the result

$\sum_{j=0}^{m}(2 j+1) N_{m}^{(j)}=3^{m}$,

as required for the number of independent components in the rank $m$ tensor.

Representations of tensors with partial index symmetry are obtained, following the usual rules for angular momentum coupling, from the appropriate nonsymmetric and fully-symmetric tensors of lower rank. For example $T_{1(2)}$ has an irreducible representation obtained by coupling the results for the vector $\boldsymbol{T}_{1}$, (three components, weight 1 ) with the index-symmetric traceless second rank tensor $T_{(2)}$ (six compo- 
nents, weights 0 and 2);

$D^{(1-)} \otimes\left\{D^{(0+)} \oplus D^{(2+)}\right\}=2 D^{(1-)} \oplus D^{(2-)} \oplus D^{(3-)}$

giving $(2 \times 3)+5+7=18$ independent components and no weight- 0 contribution.

For any allowed bimolecular process, it is necessary that each molecular tensor (of the appropriate rank $p$ or $q$ ) must possess at least some non-zero elements. The criterion is that the product of the irreducible representations of the initial and the final states of each molecule must be spanned by one or more tensor components. In the common case of transitions originating from a totally symmetric ground state, this reduces to a requirement that one of the irreducible parts of the tensor transforms under the same representation as the excited state. To determine the transformation properties involves mapping the irreducible representations of the full three-dimensional rotation-inversion group $\mathrm{K}$ onto the corresponding representations of the lower symmetry point group. A listing of the results for all common molecular and crystallographic point groups is given in ref. [17], in which irreducible representations $D^{( \pm)}$for weights $j=0$ to 4 are given for both even and odd parity $\#$.

\section{Acknowledgement}

One of us (AMB) gratefully acknowledges financial support from the Deutscher Akademischer Austauschdienst (DAAD).

\#I In Appendix 2 of ref. [17] there is an error in the omission of an entry (1) for $A_{2}^{\prime \prime}\left(D_{s h}\right)$ under the heading of Rank 1 ( - ).

\section{References}

[1] J. Qin, T.O. Nelson and D.W. Setser, J. Phys. Chem. 95 (1991) 5374.

[2] L. Wiedeman, M.E. Fajardo and V.A. Apkarian, Chem. Phys. Letters 134 (1987) 55.

[3] A.G. Page, S.V. Godbole and M.D. Sastry, J. Phys. Chem. Solids 50 (1989) 571.

[4] N. Pelletier-Allard and R. Pelletier, J. Luminescence 46 (1990) 217.

[5] M.E. Fajardo and V.A. Apkarıan, J. Chem. Phys. 89 (1988) 4102.

[6] J.C. White, Opt. Letters 6 (1981) 242.
[7] S.K. Peck and L.R. Hunter, Opt. Commun. 54 (1985) 12.

[8] B. Cheron, H. Kucal and D. Hennecart, J. Phys. B 23 (1990) 4281.

[9] J. Qin and D.W. Setser, Chem. Phys. Letters 184 (1991) 121.

[10] D.L. Andrews and K.P. Hopkıns, Advan. Chem. Phys. 77 (1990) 39.

[11] D.L. Andrews and M.J. Harlow, J. Chem. Phys. 78 (1983) 1088.

[12] D.L. Andrews and M.J. Harlow, J. Chem. Phys. 80 (1984) 4753.

[13] D.L. Andrews and K.P. Hopkins, J. Chem. Phys. 86 (1987) 2453.

[14] D.L. Andrews and K.P. Hopkins, J. Chem. Phys. 89 ( 1988 ) 4461.

[15] D.L. Andrews and A.M. Bittner, to be published.

[16] D.L. Andrews and N.P. Blake, J. Mod. Opt. 37 (1990) 701.

[17] D.L. Andrews, Spectrochim. Acta 46 A (1990) 871.

[18] E.A. Power and T. Thirunamachandran, Phys. Rev. A 28 (1984) 2671.

[19] J. Jerphagnon, D. Chemla and R. Bonneville, Advan. Phys. 27 (1978) 609.

[20] L.I. Gudzenko and S.I. Yakovlenko, Soviet Phys. JETP 35 (1972) 877.

[21] T.O. Nelson and D.W. Setser, Chem. Phys. Letters 170 (1990) 430.

[22] I. Last and F. George, Chem. Phys. Letters 177 (1991) 315.

[23] S. Foster, S. Felps, L.W. Johnson, D.P. Larson and S.P. McGlynn, J. Am. Chem. Soc. 95 (1973) 6578

[24] J.L. Ruebber, R.N. Wiener and C.A. Russell, J. Chem. Phys. 60 ( 1974$) 3166$.

[25] J. Tréhouse, D. Thomas, G. Nowogrocki and G. Tridet, Compt. Rend. Acad. Sc1. (Paris) C 268 (1969) 246.

[26] G.L. Zimmermann, S.J. Riviello, T.A. Glauser and J.G. Kay, J. Phys. Chem. 94 (1990) 2399.

[27] S. Hemmıngsson and B. Hohnberg, Inorg. Chem. 19 (1980) 2242.

[28] A. Saxena and N.K. Jha, J. Inorg. Nucl. Chem. 40 (1978) 1601.

[29] N. Islam, Australıan J. Chem. 26 (1973) 2371.

[30] R.W. Hipps and U. Macur, J. Phys. Chem. 91 (1987) 5218.

[31] V.E. Bondybey, Chem. Phys. Letters 109 (1984) 436.

[32] B. Weıner and Y. Öhrn, J. Chem. Phys. 83 (1985) 2965.

[33] C.S. Yannoni, R.D. Johnson, G. Meıjer, D.S. Bethune and J.R. Salem, J. Phys. Chem. 95 (1991) 9.

[34] S. Leach, M. Vervloet, A. Desprès, E. Bréheret, J.P. Hare, T.J. Dennis, H.W. Kroto, R Taylor and D.R.M. Walton, Chem. Phys. 160 (1992) 451.

[35] G. Herzberg, Molecular spectra and molecular structure, Vol. 1 (Van Nostrand, London, 1950).

[36] I.M. Gel'fand, R.A. Minlos and Z.Ya. Shapıro, Representations of the rotation and Lorentz groups and their applications (Pergamon Press, Oxford, 1963).

[37] V.V. Mikhailov, J. Phys. A 10 (1977) 147.

[38] N. Curwen and N. Lord, Math. Gaz. 73 (1989) 142. 